\title{
A Rare Presentation of a Brown Tumor on the Spinal Column Secondary to Chronic Renal Failure Managed by Dialysis
}

\author{
Henrik Ghantarchyan ${ }^{\mathrm{a}, \mathrm{c}}$, Karl Genovese ${ }^{\mathrm{b}}$, Amer Akmal ${ }^{\mathrm{a}}$, Jamshed Zuberi ${ }^{\mathrm{a}}$
}

\begin{abstract}
A brown tumor (BT) is a rare tumor composed of giant cells, and is a complication seen in secondary hyperparathyroidism related to chronic renal failure. Mediated by osteoclastic bone resorption, increasing bone turnover leads to the formation of a multinucleated giant cell tumor, also known as osteitis fibrosa cystica or BT. A BT is usually located at, but not limited to, the mandible, long bones, pelvis, and ribs. We present a case of a 40 -year-old female with a BT and a history of chronic renal failure managed by dialysis. A concurrent parathyroid adenoma was also found on imaging. A laminectomy of T8 and partial T7 - T9 fusion was completed, followed by a complete parathyroidectomy 10 days later.
\end{abstract}

Keywords: Osteoclastoma; Brown tumor; Secondary hyperparathyroidism; Chronic renal failure; Osteitis fibrosa cystica

\section{Introduction}

A brown tumor (BT) is a bony lesion that is a product of excess osteoclastic activity. Although a fair number of cases of BT have been reported since its discovery, there are 12 documented cases in the literature [1]. This occurs in cases of increased parathyroid hormone (PTH), which can be from primary, secondary, or tertiary causes.

Primary hyperparathyroidism (HPT) is a direct hyper-secretion of PTH from a parathyroid adenoma. Secondary HPT is a result of an indirect increase in PTH, usually a complication of chronic renal failure. Tertiary HPT appears in patients with longstanding secondary disease, as hyperplasia of parathyroid glands and loss of response to the level of calcium in serum [2].

Manuscript submitted December 22, 2019, accepted January 30, 2020

aSt. Joseph University Medical Center, 703 Main St., Paterson, NJ 07503, USA bSt. Joseph Family Medicine, Suite 201, 1135 Broad St., Clifton, NJ 07013, USA

${ }^{\mathrm{c}}$ Corresponding Author: Henrik Ghantarchyan, St. Joseph University Medical Center, 703 Main St., Paterson, NJ 07503, USA. Email: hghantar@sgu.edu

doi: https://doi.org/10.14740/jcs400
BT can be found in any bone, but most commonly occurs in the long bones, ribs, and pelvis [3]. In patients with chronic renal failure, dialysis is the mainstay treatment. With poor renal function, an increase in phosphate and decrease in calcium will result in a subsequent elevation of PTH. PTH-influenced increases in osteoclastic activity result in resorption of trabecular and cortical bone. In some instances, collections of osteoclasts, reactive giant cells, and hemorrhagic debris form a distinct mass, termed a BT of HPT [2]. A biopsy is required to confirm the diagnosis.

High-turnover bone disease can have disastrous outcomes, rarely seen nowadays [4]. The rate of parathyroidectomies decreased with the emergence of calcimimetics [4].

\section{Case Report}

A 40-year-old Hispanic female with a history of chronic renal failure managed by dialysis for 10 years presented to the emergency department with complaints of pain located at the abdomen and mid-back. With a recent hernia repair, the patient was thought to have symptoms of wound dehiscence. However, a non-contrast computed tomography (CT) (Fig. 1) revealed an incidental finding of a lytic lesion in the spinous process of T8 extending into the spinal canal causing apparent cord compression.

The patient had a significant amount of compression of the cord, but neurologically with minimal weakness in lower extremities. A bone biopsy revealed spindle cells with abundant osteoclast-like giant cells. These histological findings may represent a BT of HPT, which may be secondary to end-stage renal disease $(\mathrm{ESRD})$. A $1.0 \times 1.2 \times 1.4 \mathrm{~cm}$ hyper-vascular lesion was also seen along the right tracheoesophageal groove on CT cervical spine, compatible with a parathyroid adenoma. A laminectomy was recommended of T8 and partial T7 - T9 fusion operation to stabilize the area. The entire tumor and all the pieces were resected in entirety including the pedicles. The spinal cord was compression free once the tumor was resected. Ten days later, a parathyroidectomy was completed, in which all four lobes of the parathyroid were removed. The patient had a stable discharge from the hospital and was instructed to follow up with the primary care physician 5 days postoperative parathyroidectomy. In the follow-up visit, patient was free of neurological deficits or symptoms of hyper or hypocalcemia. 


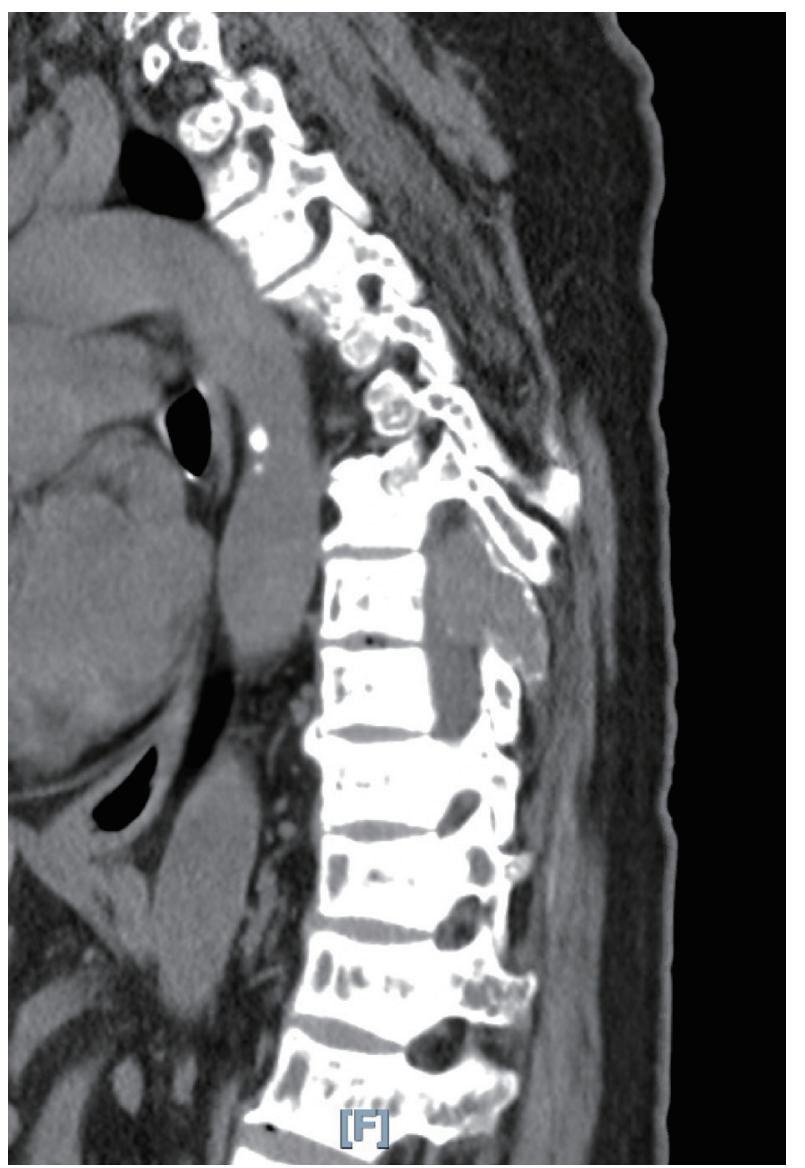

Figure 1. Characteristic CT image of the tumor. CT: computed tomography.

\section{Discussion}

In patients with chronic kidney disease treated with dialysis, BT is a complication of secondary HPT. This commonly in-

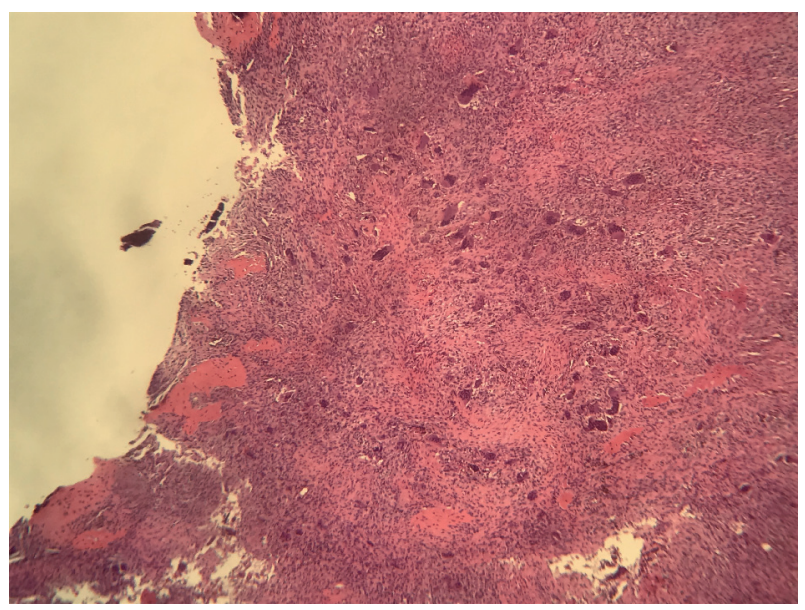

Figure 2. Low-power view shows numerous osteoclast-like multinucleated giant cells embedded in sheets of fibroblasts. Ossification foci are at the periphery (top left).

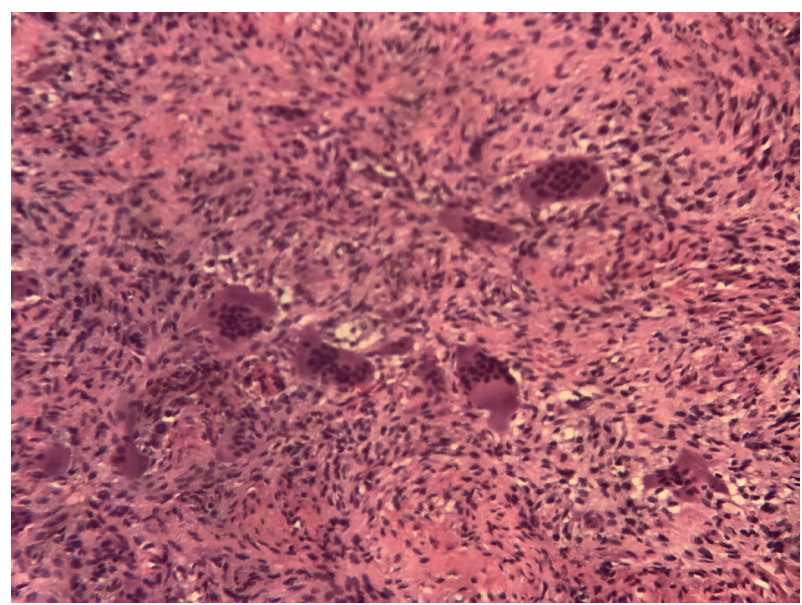

Figure 3. Medium-power view shows osteoclast-like multinucleated giant cells. Hemosiderin deposition is noted in the background.

volves the mandible, ribs, and long bones [5]. The importance of the prompt diagnosis of the BT is to establish a multidisciplinary management to prevent progression, neurologic complications and sequelae (radiculopathy and myelopathy) despite its slow progression and certainly benign behavior [6].

Medical intervention of normalizing blood calcium and phosphate levels should stop the progression of a BT. If unsuccessful, a total or subtotal parathyroidectomy is recommended [7]. Our patient was initially managed pharmacologically with cinacalcet, but responded insufficiently. Upon literature review, a study among 73 hemodialysis patients, whose bones were periodically checked, showed three asymptomatic BTs within 5 years after starting dialysis (PTH varied between 5 and 15 times the upper limit) [8].

The mechanism of secondary HPT leading to a BT is caused mainly by an increase in phosphate, decrease in ionized calcium, and a decrease in calcitriol. With a poor glomerular filtration rate (GFR), the phosphate excretion by the kidneys is at a minimum, thus leading to elevated phosphate levels. Increased phosphate levels further contribute to the formation of parathyroid mass by increasing the synthesis of fibroblast growth factor 23 (FGF-23) by stimulation of PTH. Elevated levels of FGF-23 can further suppress calcitriol levels, which can result in decreased ionized calcium. Due to the diminished activity of the kidney, the final and active form of vitamin D, calcitriol, will be decreased. With low levels of calcitriol, there will be a decrease in mobilization of calcium and phosphate from the gut, and decreased calcium resorption in the kidney, thus leading to increased levels of PTH. Additionally, because PTH is metabolized in the liver and the kidney, decreased renal function of ESRD may be responsible for increased serum levels of PTH due to slowed catabolism [9]. Increased PTH secretion and expression of numerous other factors (such as interleukin-1 (IL-1), tumor necrosis factor- $\alpha$ (TNF- $\alpha$ ), IL-6 and IL-2) incite bone resorption which results in osteopenia [10].

Upon histologic evaluation, sheets of fibroblasts are seen alongside numerous osteoclast-like multinucleated giant cells (Fig. 2). Scattered foci of bone production and resorption are also seen [9]. Another characteristic feature of this lesion is the 
presence of fresh hemorrhage and brown colored hemosiderinladen macrophages (Fig. 3).

With an increase in dialysis-dependent patients, an increase in BT prevalence can also be expected. Management of secondary HPT can be achieved with conventional methods, such as a diet low in phosphate and pharmacological methods, such as phosphate binders (sevelamer). In the case presented, the patient presented to the hospital with clinical manifestations of secondary HPT 1 year prior. As a total parathyroidectomy was recommended, the surgical procedure was not followed through. The patient presented 1 year later with worsening clinical symptoms and additional neurological findings.

If conventional and pharmacological methods are seen to provide minimal benefit, a total or subtotal parathyroidectomy is recommended. Although parathyroidectomy is curative, surgical removal of the BT mass may sometimes be required [11]. In the modern era, parathyroidectomy is mainly reserved for severe unremitting HPT refractory to medical treatment, and associated with severe hypercalcemia, progressive and debilitating hyperparathyroid bone disease, refractory pruritus, progressive extra-skeletal calcification or calciphylaxis and otherwise unexplained myopathy [4]. With an unusual presentation on the spinal column as seen in this case, clinical manifestations are noticed at later stages due to a mass effect of cord compression.

In patients with chronic renal failure, neurologic manifestations, even in the mildest form, should raise suspicion of a BT. Inspection in the common locations for a BT should include the long bones, pelvis, and mandible in addition to the spinal cord, when supported by the clinical presentation. Given elevated PTH levels, we recommend a total or subtotal parathyroidectomy in patients presenting with secondary HPT.

\section{Conclusions}

We present a 40-year-old female patient with chronic renal failure treated with dialysis who presents with an osteoclastic BT located on the spinal column. This case serves as an example of the importance of a suggested total parathyroidectomy in a patient with chronic renal failure and secondary HPT to prevent the progression of a BT.

\section{Acknowledgments}

None to declare.

\section{Financial Disclosure}

This research did not receive any specific grant from funding agencies in the public, commercial, or not-for-profit sectors.

\section{Conflict of Interest}

None to declare.

\section{Informed Consent}

Not applicable.

\section{Author Contributions}

$\mathrm{KG}$ and $\mathrm{HG}$ participated in the patient care. $\mathrm{HG}$ wrote the case report. JZ, KG, and AA revised and edited the case report.

\section{Data Availability}

The authors declare that data supporting the findings of this study are available within the article.

\section{References}

1. Alfawareh MD, Halawani MM, Attia WI, Almusrea KN. Brown tumor of the cervical spines: a case report with literature review. Asian Spine J. 2015;9(1):110-120.

2. Jakubowski JM, Velez I, McClure SA. Brown tumor as a result of hyperparathyroidism in an end-stage renal disease patient. Case Rep Radiol. 2011;2011:415476.

3. Silva MT, Cedraz JS, Pontes CG, Trento CL, Brasileiro BF, Piva MR, Pereira FA. Brown tumor: clinical findings of secondary hyperparathyroidism in patients with renal osteodystrophy. Gen Dent. 2017;65(2):70-74.

4. Rodrigues N, Godinho I, Leite Nogueira E, Jorge S, Lopez-Presa D, Gomes da Costa A, Weigert AL. Multiple brown tumors - a rare presentation in the modern era. Clin Nephrol. 2018;89(1):57-60.

5. Liu YJ, Frauenhoffer EE, Walker E, Williams NC. A case report of brown tumor in a patient with chronic renal failure and renal cell carcinoma. Diagn Cytopathol. 2018;46(4):355-361.

6. Sanchez-Calderon MD, Ochoa-Cacique D, Medina Carrillo O, Garcia Gonzalez U, Vicuna Gonzalez RM, Bravo Reyna CC, Guerra-Mora JR. Brown tumor of the cervical spine in a patient with secondary hyperparathyroidism: A case report. Int J Surg Case Rep. 2018;51:328-330.

7. Lee S, Lerer DB, Dorfman HD, Coco M. Brown tumors developing in renal transplant recipients with persistent hyperparathyroidism: two case reports and review of literature. Clin Nephrol. 2004;61(4):289-294.

8. Kampschreur LM, Hoogeveen EK, Op den Akker JW, Beutler JJ, Beems T, Dorresteijn LDA, de Sevaux RGL. A haemodialysis patient with back pain: brown tumour as a cause of spinal cord compression under cinacalcet therapy. NDT Plus. 2010;3(3):291-295.

9. Fineman I, Johnson JP, Di-Patre PL, Sandhu H. Chronic renal failure causing brown tumors and myelopathy. Case report and review of pathophysiology and treatment. J Neurosurg. 1999;90(2 Suppl):242-246.

10. Mourelatus Z, Goldberg H, Sinson G, Quan D, Lavi E. 
Case of the month: March 1998 - 48 year old man with back pain and weakness. Brain Pathol. 1998;8(3):589590.

11. Fatma LB, Barbouch S, Fethi BH, Imen BA, Karima
$\mathrm{K}$, Imed $\mathrm{H}$, Fethi el $\mathrm{Y}$, et al. Brown tumors in patients with chronic renal failure and secondary hyperparathyroidism: report of 12 cases. Saudi J Kidney Dis Transpl. 2010;21(4):772-777. 